\title{
Genetic Algorithm Based Improved ESTAR Nonlinear Models for Modelling Sunspot Numbers and Global Temperatures
}

\section{Bishal Gurung}

Royal University of Bhutan

Achal Lama ( $\nabla$ achal.lama@icar.gov.in )

Indian Agricultural Statistics Research Institute https://orcid.org/0000-0002-5376-3760

\section{Santosha Rathod}

ICAR-Indian Agricultural Statistics Research Institute

K N Singh

Royal University of Bhutan

\section{Research Article}

Keywords: Smooth Transition Autoregressive model, Non-linear model, Sunspot numbers, Global mean temperatures, Optimal out-of-sample forecast

Posted Date: August 17th, 2021

DOI: https://doi.org/10.21203/rs.3.rs-695500/v1

License: (c) (i) This work is licensed under a Creative Commons Attribution 4.0 International License. Read Full License 
Genetic Algorithm based improved ESTAR nonlinear models for modelling sunspot numbers and global temperatures

\author{
Bishal Gurung ${ }^{1,2}$, Achal Lama $^{2 *}$, Santosha Rathod ${ }^{3}$ and K N Singh ${ }^{2}$ \\ ${ }^{1}$ Sherubtse College, Royal University of Bhutan, Bhutan \\ ${ }^{2}$ ICAR-Indian Agricultural Statistics Research Institute, New Delhi, India \\ ${ }^{3}$ ICAR-Indian Institute of Rice Research, Hyderabad, India
}

*Corresponding Author: Achal Lama, achal.lama@icar.gov.in,ORCID ID: 0000-0002-5376-3760 


\begin{abstract}
Smooth Transition Autoregressive (STAR) models are employed to describe cyclical data. As estimation of parameters of STAR using nonlinear methods was time-consuming, Genetic algorithm (GA), a powerful optimization procedure was applied for the same. Further, optimal one step and two step ahead forecasts along with their forecast error variances are derived theoretically for fitted STAR model using conditional expectations. Given the importance of the issue of global warming, the current paper aims to model the sunspot numbers and global mean temperatures. Further, appropriate tests are carried out to see if the model employed is appropriate for the datasets.
\end{abstract}

Keywords Smooth Transition Autoregressive model • Non-linear model- Sunspot numbers. Global mean temperatures - Optimal out-of-sample forecast

\title{
1. Introduction
}

Linear time-series family of models viz., Autoregressive integrated moving average (ARIMA), are useful for modelling and forecasting of time-series datasets in order to extract substantial statistics and other characteristics of the data. It takes into consideration that data points taken over different time-epochs may have internal structure, like, autocorrelation, trend or seasonal variation. A time series model is linear if it can be written as a linear function of past observations, errors and other exogenous variables. These linear models have gained much popularity as it is relatively simple and further there exist a good number of computer software which has inbuilt packages for fitting such models. The linear model, however, is inadequate as it is not capable of capturing many important characteristic features like presence of nonGaussian colored noise in the error term, cyclicity, nonlinearity, limit cycle behavior, volatility clustering, leverage effects, and chaotic behavior of time-series datasets. These types of characteristics feature of time-series datasets can be modelled efficiently using various nonlinear time series models available in the literature. Keeping this in mind, time-series analysis has moved towards the nonlinear domain over the last four decades, as it not only provides a better fit to the data but is also capable to capture different features which cannot be captured solely by linear models. Further, nonlinear models are more suitable for accurately relating dynamics of a time-series, and for making better multi-step-ahead forecasts. To this end, to capture cyclical phenomena in time-series data, Smooth Transition Autoregressive (STAR) 
family of parametric nonlinear time-series models was propounded by Terasvirta (1994). There are mainly two models under the STAR family, Exponential Smooth Transition Autoregressive (ESTAR) and Logistic Smooth Transition Autoregressive (LSTAR).

The advantages of using STAR models can be gauge from the fact that these types of models are of particular importance to describe those data sets that have cyclical variations along with chaotic periods, changes in economic aggregates influenced by changes in the manners of many different agents and it is highly unlikely that all agents react simultaneously to a given economic signal. In STAR models, transitions are possible along a continuous scale, making the regimeswitching process smooth which helps overcome the abrupt switch in parameter values characteristic of simpler Threshold autoregressive (TAR) models.

The importance of precise modelling of climatic variables such as temperature and sunspots numbers can be judged from the fact that researchers have used variety of models ranging from parametric to nonparametric and recently machine learning too (Citakoglu et al. 2020, Gurung et al. 2021, Merkenschlager et al. 2021, Taki et al. 2021,). We, in this study have proposed efficient Genetic algorithm (GA) based ESTAR model as an alternative to efficiently model and forecast the very important climatic factors sunspots numbers and global temperatures.

In this manuscript, after a brief introduction in Section 1, Section 2 describes ESTAR models in detail. In Section 3, a brief narrative on fitting of star models is given. In Section 4, optimal one step and two step ahead forecasts of ESTAR model is theoretically derived. Section 5 illustrates the procedure with practical time-series datasets. Finally, in Section 6 some concluding remarks are put up and the papers ends with delineation of some pertinent research problems for future work.

\section{Description of ESTAR Model}

Over the last few decades, nonlinear time-series models have been applied in many fields of research. One such parametric family is the State dependent models (SDM), which are the general class of nonlinear models that include the Bilinear and Threshold autoregressive as

special cases, but allow much greater flexibility. SDM are essentially Autoregressive moving average (ARMA) models in which the parameters are functions of past values of time-series 
(Young et al., 2001). Although SDM are of a general nature, they are nevertheless amenable to statistical analysis. This approach to nonlinear time-series analysis offers two major advantages, namely

(i) SDM may be used directly in connection with the problem of forecasting.

(ii) Since these can be fitted to data without any specific prior assumptions about the form of the nonlinearity, these may be used to give us an "overview" of the nonlinearity inherent in the data, and thus indicate whether, for example, a Bilinear, Threshold autoregressive, or even a linear model, is appropriate.

Toivonen (2003) derived the discrete-time models with state-dependent parameters for nonlinear systems. Linearization followed by integration over the sampling interval was used to represent these systems. The representation gave exact solution of the nonlinear system. Numerical simulations also showed that these models could be represented by a neural network approximator trained on input-output data. Models which allow for state-dependent or regimeswitching behavior have been most popular for such analysis. One of the most popular models with regime-switching behavior is Threshold Autoregressive (TAR) models. The foremost thought of TAR models is to model a given stochastic process by a piecewise linear autoregressive model, where the determination as to whether each of the sub-models is active or not is driven by the value of a known variable. Smooth Transition Autoregressive (STAR) family of parametric nonlinear time-series models is a generalization of the threshold models, avoiding discontinuities in the autoregressive parameters as the transition which takes place from one regime to the next is determined by a continuous nonlinear function.

It is to be noted that STAR family of parametric nonlinear time-series model has the potential to capture the non-Gaussian characteristics at different time-epochs of time-series datasets. The STAR family of models can be written as:

$$
y_{t}=G\left(z_{t}, y_{t} ; \boldsymbol{\Psi}\right)=\boldsymbol{\varphi}^{\prime} \boldsymbol{w}_{\boldsymbol{t}}+\left(\boldsymbol{\theta}^{\prime} \boldsymbol{w}_{\boldsymbol{t}}\right) G_{k}^{L}\left(\gamma, c ; y_{t-d}\right)+\varepsilon_{t}
$$

where $\left\{\varepsilon_{t}\right\}$ is a sequence of normal $\left(0, \sigma^{2}\right)$ independent errors, $\boldsymbol{\varphi}=\left(\phi_{0}, \phi_{1}, \ldots, \phi_{p}\right)^{\prime}$ and $\boldsymbol{\theta}=$ $\left(\theta_{0}, \theta_{1}, \ldots, \theta_{p}\right)^{\prime}$ are $(p+1) \times 1$ parameter vector, $\boldsymbol{w}_{\boldsymbol{t}}=\left(1, y_{t-1}, \ldots, y_{t-p}\right)^{\prime}$ is the vector consisting of an intercept and the first $\mathrm{p}$ lags of $y_{t}$ and $G_{k}^{L}\left(\gamma, c ; y_{t-d}\right)$ is known as transition function which 
determines how the regime changes. The way the autoregressive coefficients behave is essentially constant in each of the regimes with a continuous smooth change between regimes, whose rate of change is controlled by the constant $\gamma_{L}(>0)$. Self-Exciting Threshold Autoregressive (SETAR) models, Exponential Smooth Transition Autoregressive (ESTAR) models, logistic smooth transition autoregressive (LSTAR) models are the special cases of STAR model.

There are two different transition functions in the smooth transition autoregressive models, one is

$$
G\left(y_{t-d}\right)=\left(1+\exp \left[-\gamma\left(y_{t-d}-c\right)\right]\right)^{-1} \quad \gamma>0
$$

And the other one

$$
G\left(y_{t-d}\right)=1-\exp \left(-\gamma\left(y_{t-d}-c\right)^{2}\right) \quad \gamma>0
$$

$G\left(y_{t-d}\right)$ is bounded between 0 and 1 , which realizes the smooth transition between regimes dynamically rather than a unexpected jump from one regime to the other, $\mathrm{c}$ is the threshold value and parameter $\gamma$ determines the speed and smoothness of the transition. In Eq. 3 when

$$
\gamma \rightarrow \infty \text {, if } y_{t-d} \leq c \text { then } G\left(y_{t-d}\right)=0, \text { and if } y_{t-d}>c \text { then } G\left(y_{t-d}\right)=1
$$

which means Eq. 2 can be taken as a TAR (p) model. Further, when $(\gamma \rightarrow 0), G\left(y_{t-d}\right)$ becomes a linear AR (p) model. So model (1) with transition function (2) is called the LSTAR model, and with transition function (3) is called the ESTAR model. A tiny difference in the two transition functions is owing to the fact that, the logistic function changes monotonically with $y_{t-d}$, while the exponential function fluctuates symmetrically at $\mathrm{c}$ with $y_{t-d}$. But, it is to be kept in mind that both the functions become steeper when $\gamma$ is large, which means more rapid is the speed of the transition. One of the characteristic features of (1) is that the minimum value of the transition function can be equal to zero. Also heartening to note that other linear as well as nonlinear models can be transformed as special cases of the STAR specifications. The coefficients for lagged values of $y_{t}$ changes smoothly with $y_{t-d}$, which is determined by the main function of the transition function $G_{k}^{L}\left(\gamma, c ; y_{t-d}\right)$, in a way allowing the local dynamics of the model to change with respect to $y_{t-d}$. The local dynamics as a function of a lagged value of $y_{t}$ is modelled to study the nonlinear effects of a shock. If a shock of a negative type pushes a characterization away from a locally stable regime, the change in the value of transition function 
subsequently changes the local dynamics. If the regime contains a pair of explosive complex roots, $y_{t}$ may be returned to the previous level a lot more speedily than would be the case, if it had followed a linear AR process. Another characteristic feature of the transition function $G$ of ESTAR model is that it is symmetric about c. The implication of which is, that the local dynamics are similar for high as well as for low values of $y_{t-d}$, while the behaviour of the variable is a tad different for the mid-range. Moreover, the transition function for ESTAR allows $y_{t}$ to shift smoothly between very small and very large values for which local dynamics are stable.

\subsection{Fitting of STAR models}

The above STAR family of nonlinear time-series models cannot be estimated by the 'Method of least squares' as minimization of residual sum of squares yield normal equations which are nonlinear in the parameters. Since it is not possible to solve nonlinear equations exactly, the next alternative is to obtain approximate analytic solutions by employing iterative procedures, viz., Linearization method, Steepest Descent method and Levenberg-Marquardt's method. The details of these methods along with their merits and demerits can be read from Draper and Smith (1998). In linearization method, the result of linear least square theory is employed in a sequence of stages. However, this methodology along with the steepest descent is not ideally suited for parameter estimation as it converges to true parameter values even if initial trial values are far-off from the true parameter values, but this convergence tends to be very time-consuming at the later stages of the iterative process. The linearization method will not converge until and unless the vicinity of the true parameter values is reached, but if initial guess values of the

parameters are too far away, convergence may not occur at all. The third and the most popularly used method of computing nonlinear least squares estimators is the Levenberg-Marquardt's method. This method takes into account the best features of both and avoids their serious lacunae. It is good in the sense that it almost always converges and does not 'slow down' at the latter part of the iterative process. All the above three procedures for parameter estimation requires initial values of the parameters and the choice of good initial values is very crucial. However, there is no standard course of action for getting initial estimates. The most obvious method for making initial guesses is by the use of prior information. Estimates obtained from experiments conducted before, known values for analogous experiments, values calculated from 
theoretical basis all these form ideal initial guesses. Some other methods are Linearization, solving a system of equations, using properties of the model, Graphical method. Popularity of these methods is certainly due to their relative simplicity, robustness and also due to the existence of computer software incorporating the same. Nowadays most of the standard statistical packages contain computer programmes to fit nonlinear statistical models based on Levenberg-Marquardt algorithm. For example, SPSS has NLR option, SAS has NLIN option, and IMSL has RNSSQ option to accomplish the task.

However, for fitting the ESTAR model we tried using different nonlinear techniques in SAS 9.4 and R software. But even two hours of waiting for program to provide output in the form of estimated parameters and predicted value of observations turned futile. Keeping this in mind, we employed the Genetic algorithm (GA) methodology for parameter estimation. GA is a technique used for optimization which is based on natural selection of the fittest individuals. GA repeatedly modifies a population of individual solutions. At each step, GA chooses individuals arbitrarily from the population to be parents and employs them to produce the children for the next generation. Over repeated generations, the population "evolves" en route the optimal solution of optimization. GA uses three main types of rules at each step to create the next generation from the current population. A detailed description is given below:

- Step1: The Initial population consisting of several individuals

- Step2 : A number of individuals from a different population are mixed to original population to maintain diversity

- Step3 : Crossover and mutation operations are applied to the individuals

- Step4 : Fitness values of all individuals are calculated by NN model

- Step5 : Superior individuals are selected and retained for next generation

- Step6 : step 2 through 5 are repeated until an arbitrary condition satisfied.

Using the GA methodology, $\mathrm{Wu}$ and Chang proposed a method to estimate the threshold and lag parameters for TAR models. Baragona et al.(2004) estimated the parameters and threshold values of subset autoregressive moving average model by making use of GA methodology. 


\section{An illustration}

The data are at monthly frequency for global land-ocean temperatures (GT) and sunspot numbers (SS), and cover the period from January 1971 to February, 2018. In terms of the data, the global temperatures were obtained from the National Aeronautics and Space Administration's (NASA), Goddard Institute for Studies (GISS), while the sunspot numbers were obtained from the Solar Influences Data Analysis Centre. Woodward and Gray proposed new ARMA models for the yearly sunspot numbers. It was shown to be superior to the well-known ARMA $(2,0)$ model suggested by the Box-Jenkins approach. From the total 566 data points denoted as $\left\{\boldsymbol{X}_{t}, t=\right.$ $0,1, \ldots, 565\}$, first 500 data points corresponding to frequency for global land-ocean temperatures (GT) and sunspot numbers for the period 1971 to 2012 are used for building the model and remaining 66 data points for validation purpose. The entire data analysis is carried out using SAS (9.4) and R 3.4.2 software packages.

A directed scatter diagram at lag- $k$ is a powerful graphical tool (Fan and Yao, 2003) in analyzing nonlinear time series.

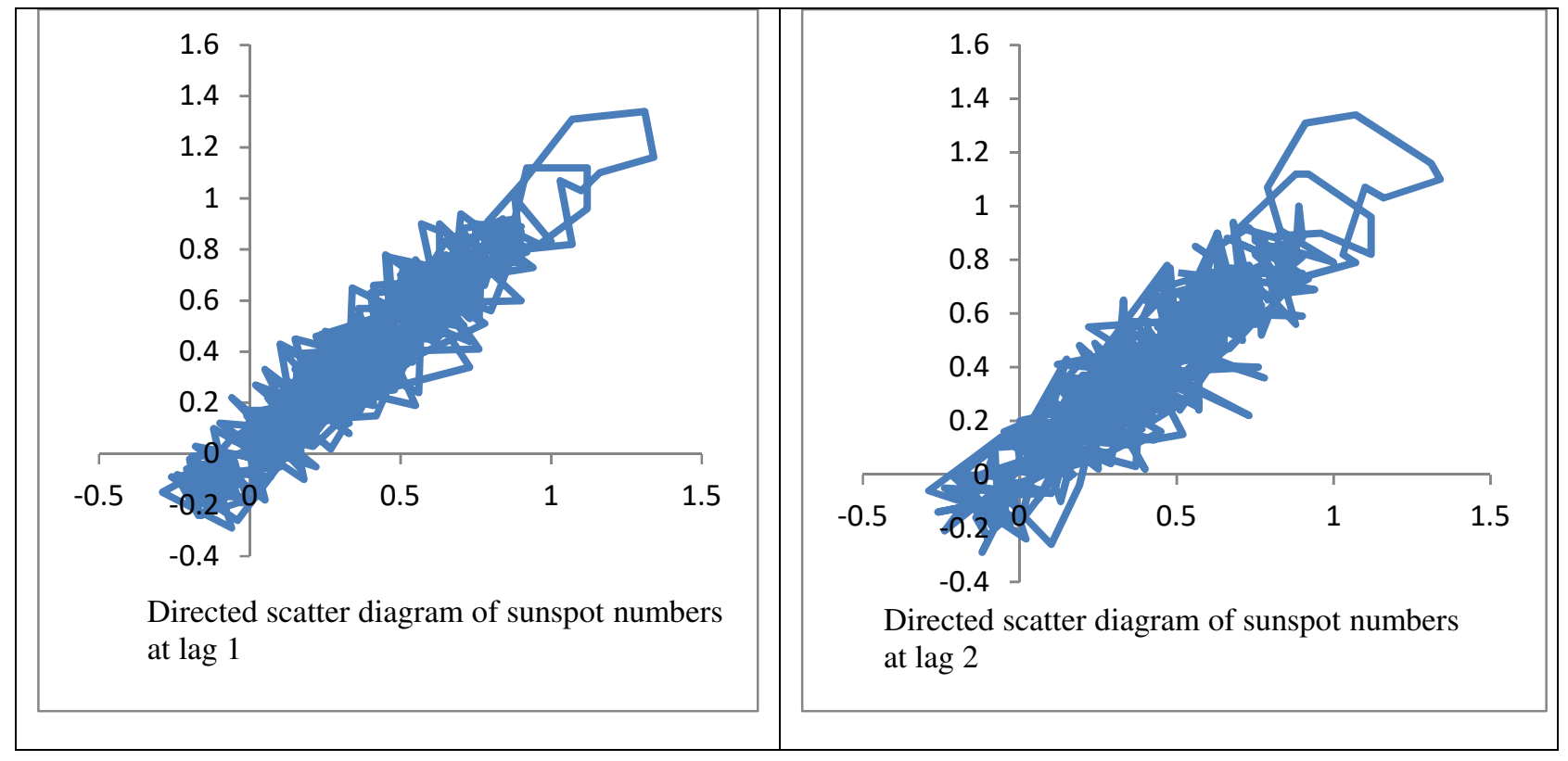




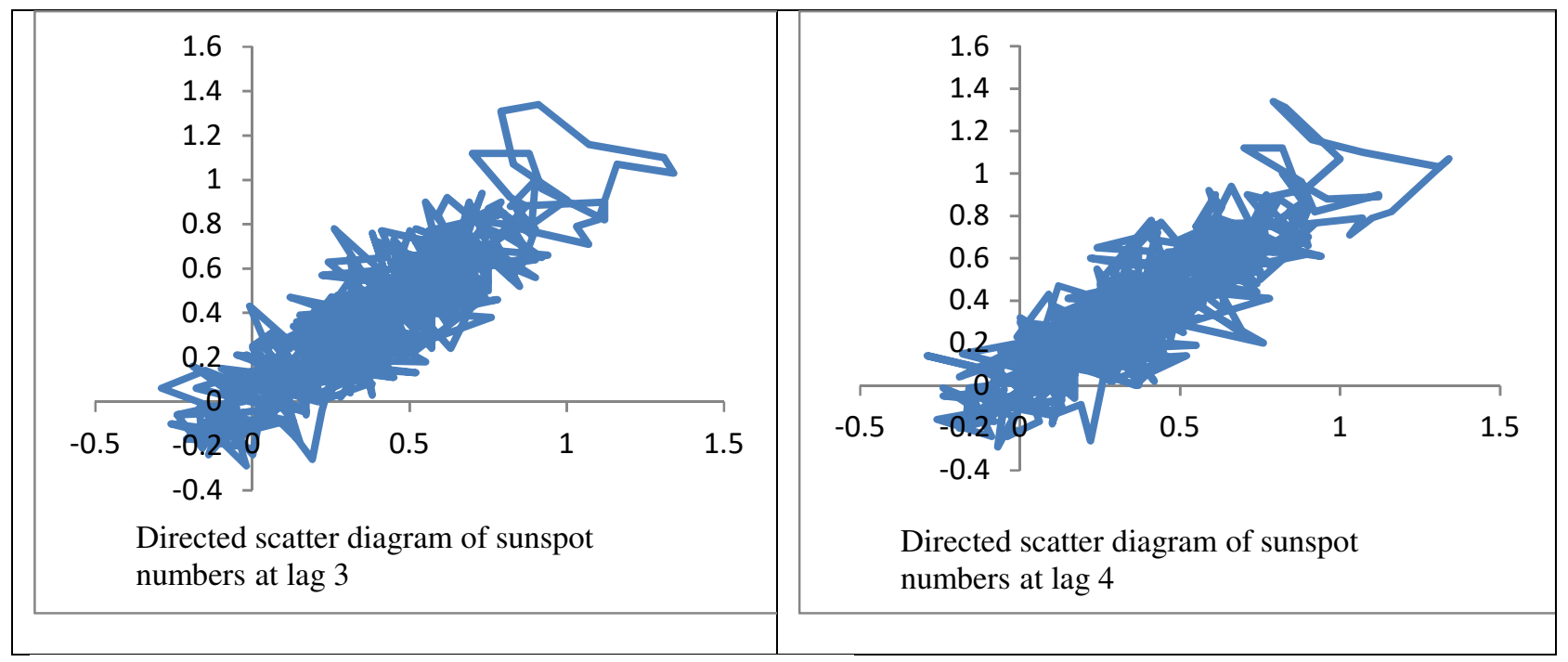

Fig. 1a Directed scatter diagram of sunspot numbers

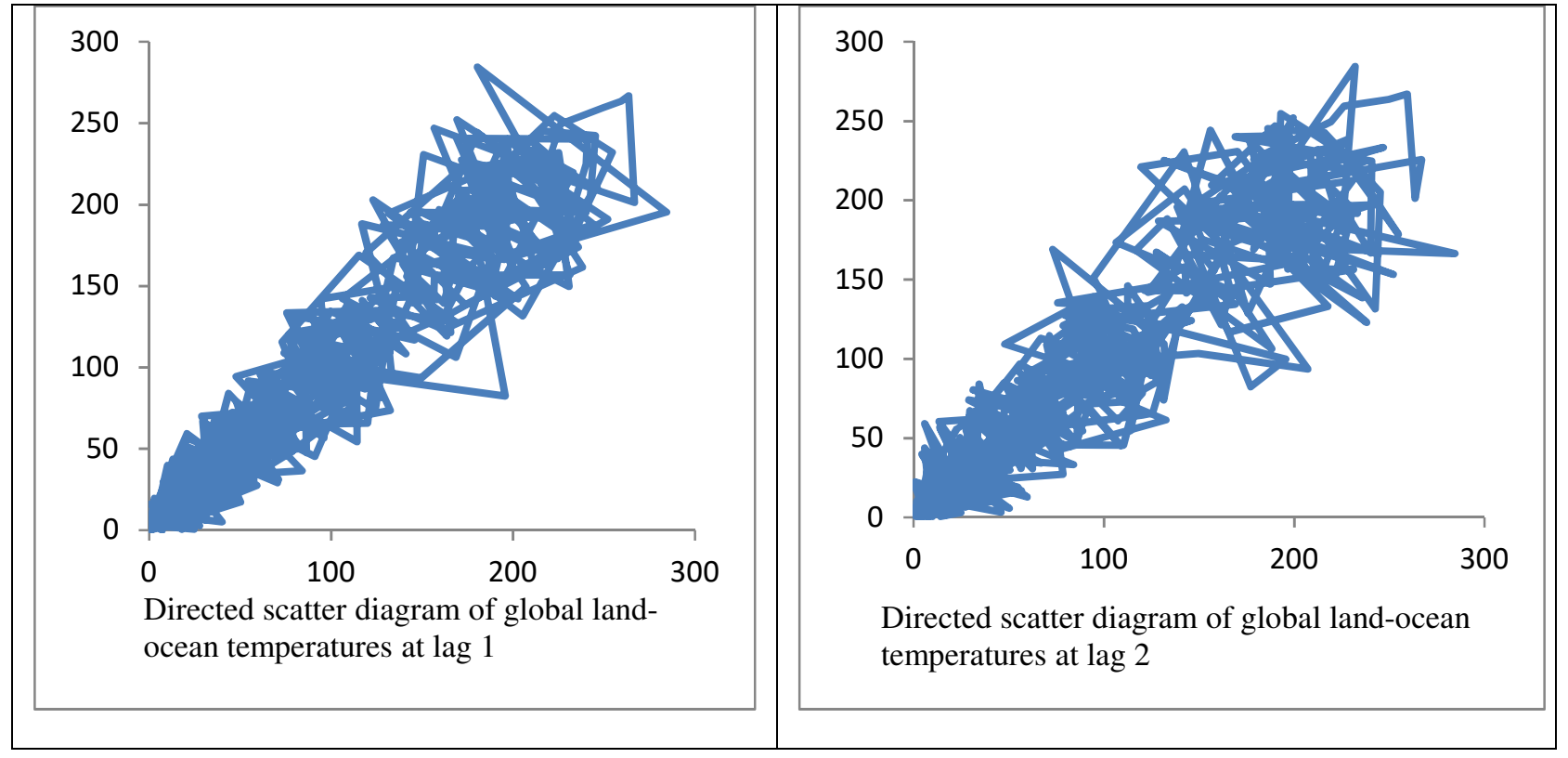




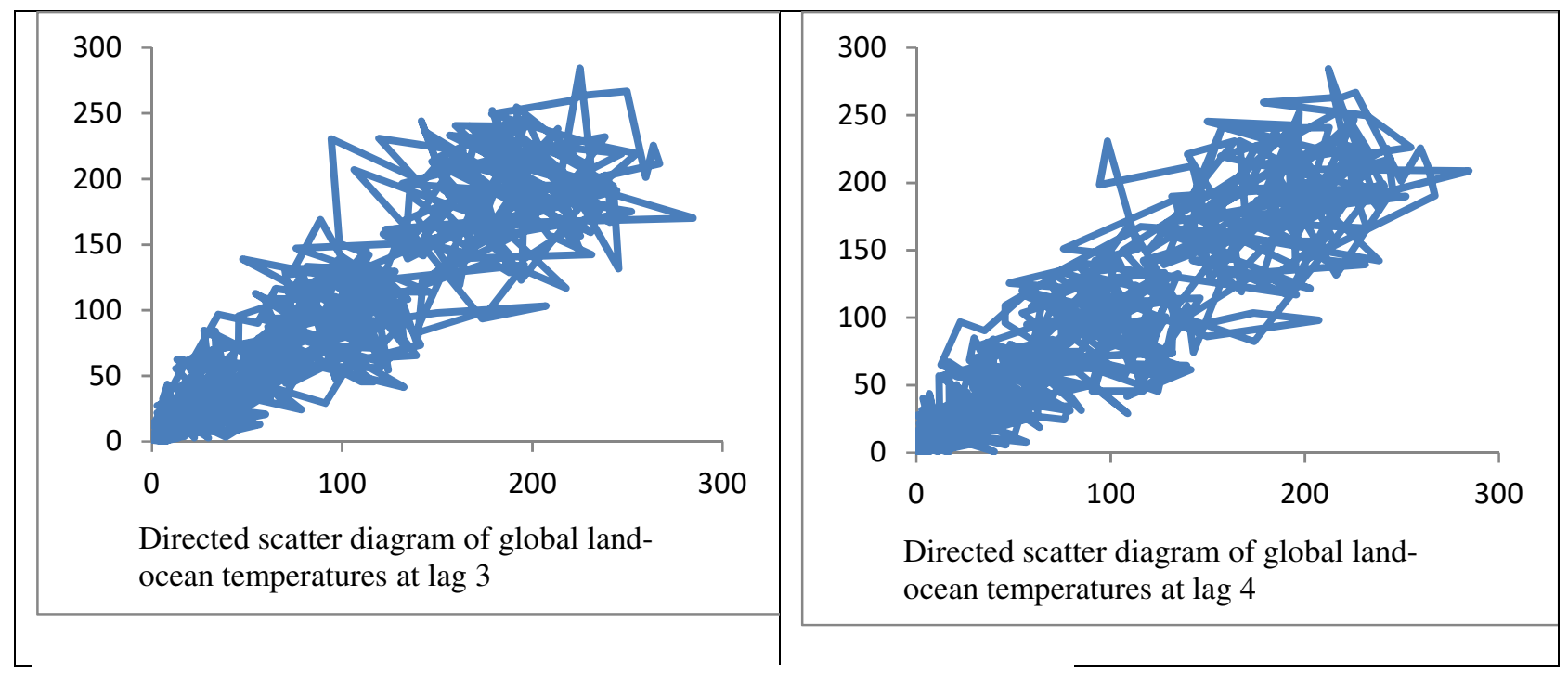

Fig. 1b Directed scatter diagram of global land-ocean temperatures

From the above figures, it is seen that there is a void in the directed scatter plot at various lags which point credibly that the joint distribution of $\left(X_{t}, X_{t-i}\right)$ is not Gaussian since a stationary Gaussian process cannot have void in a scatter plot. The characteristic feature of scatter plots with void indicates presence of limit cycle behavior of above cyclical data. Further, graph of the landings data in the conventional and the reversed time order exhibit a periodic-like fluctuation. The time-series data do not show similar look while progressing in the forward and backward directions with respect to time. This indicates that the statistical properties of the data are not invariant under reversibility of time direction. Therefore, the time-series data are not realization of static transformation of a linear Gaussian random process. Further, it was also found that the periodogram ordinates were significant, thereby signifying presence of cyclicity. Obviously, conventional ARIMA modelling approach of the given two time-series data may not be able to describe these datasets satisfactorily.

The preliminary data analysis justifies the application of ESTAR nonlinear time-series model to describe the two time-series dataset. Accordingly, several ESTAR models were fitted to the data and the best model was identified on the basis of minimum AIC criterion. The best ESTAR models for global land-ocean temperatures and sunspot numbers are respectively obtained as 


$$
\begin{gathered}
Y_{t}=1.098764+0.689453 Y_{t-1}+0.26952 Y_{t-2}-0.86148 Y_{t-3} \\
+\left[5.946853+1.170439 Y_{t-1}-0.27681 Y_{t-2}+2.683797 Y_{t-3}\right][1 \\
\left.-\exp \left\{-0.06181\left(y_{t-3}-1.639076\right)^{2}\right\}\right] \\
Y_{t}=-8.25914-0.71171 Y_{t-1}+11.39342 Y_{t-2} \\
+\left[12.52122+1.402113 Y_{t-1}-11.135 Y_{t-2}\right][1 \\
\left.-\exp \left\{-0.868706\left(y_{t-2}-2.022293\right)^{2}\right\}\right]
\end{gathered}
$$

To get a visual idea, fitted ESTAR models along with data points are exhibited in Figures 2.12.2. A perusal of the graphs shows that fitted ESTAR models have captured properly the cyclical behavior present at various time epochs for both the datasets under consideration.

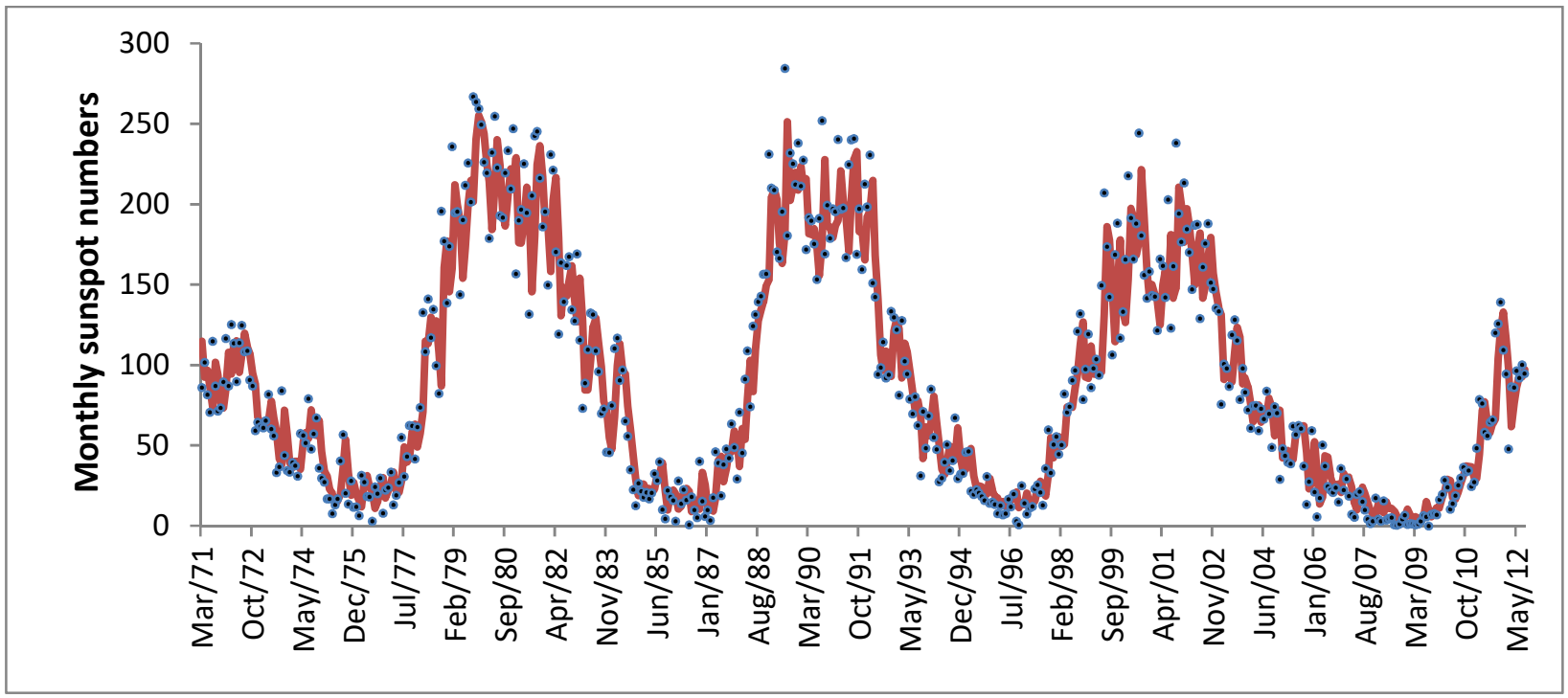

Fig 2a Fitted ESTAR model along with monthly sunspot numbers data 


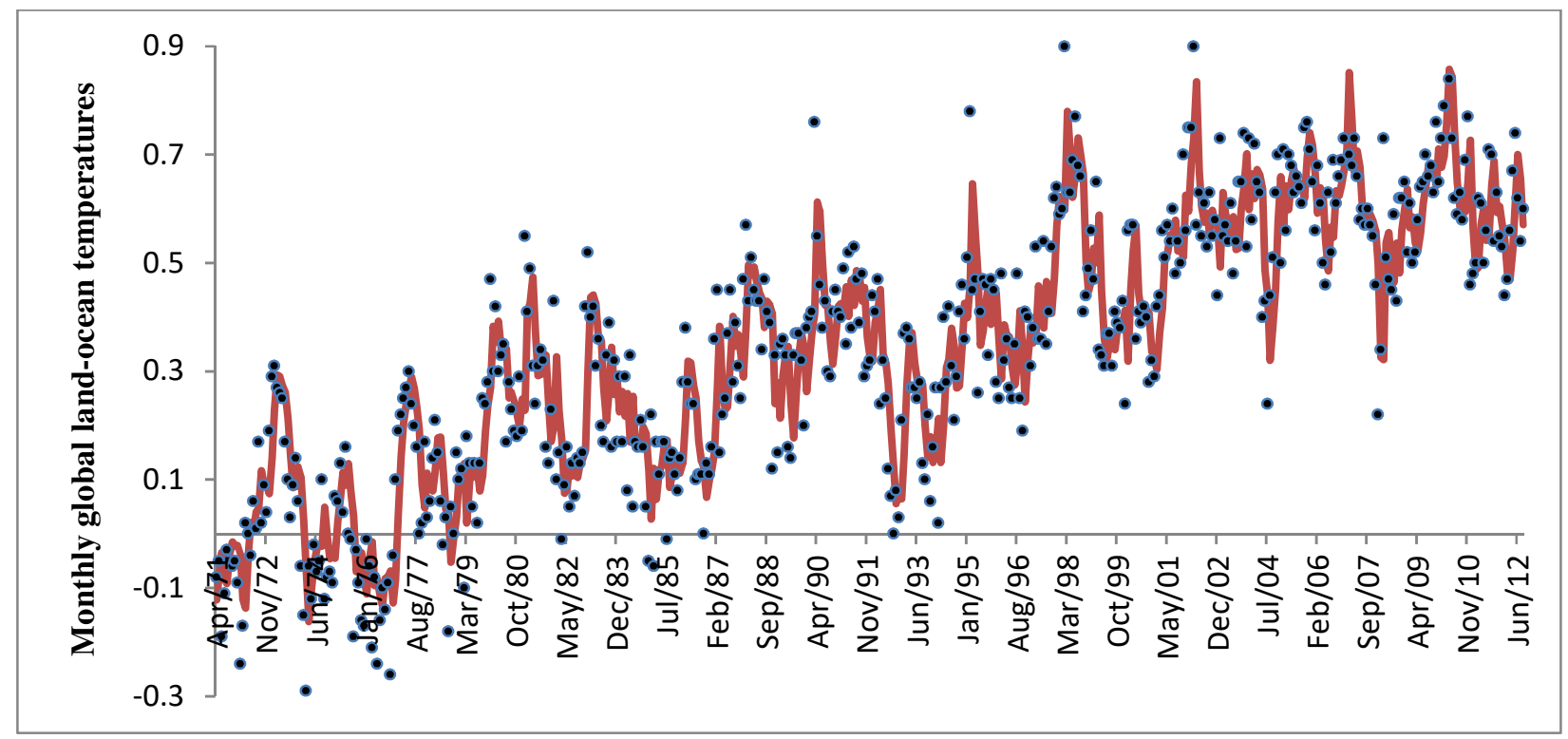

Fig 2b Fitted ESTAR model along with monthly global land-ocean temperatures data

Detailed analysis of the residuals is strongly recommended to decide about the suitability of a model. Three important assumptions made in the model are:

$>$ Errors are independent

$>$ Errors are normally distributed.

Error variances are constant(Homoscedastic)

These assumptions can be verified by examining the residuals. If the fitted model is correct, the residuals should exhibit tendencies that tend to confirm or at least should not exhibit a denial of the assumptions. Further, the values of Ljung-Box Q are calculated, using maximum lag as 20, and they are all found to be less than the tabulated values thereby indicating that the fitted ESTAR models are properly specified. Further, from the Run test we can see that the p value is more than 0.01 that means test is not significant and the null hypothesis is accepted (errors are independent) which means that there is nothing more in the data which is not explained. Also, the graph between residuals and $\mathrm{p}(\mathrm{x})$ is almost a straight line which means residuals are following normal distribution. We also can infer about the homoscedasticity of errors from Breusch-Pagan test tests. Taking all this into consideration, we can infer that the fitted model is appropriate for the data under consideration. 


\section{Derivation of formulae for out-of-sample forecasts}

One of the main objectives when dealing with time-series analysis is the forecasting of future values of the series of interest. In this section, we confine our attention only to deriving out-ofsample one-step and two-step forecast formulae in respect of ESTAR model. However, formulae for more than two-step ahead forecasts, though quite complicated, can be derived along similar lines. The optimal predictor which minimizes the mean one-step-ahead squared prediction error is the conditional expectation given by

$$
\begin{gathered}
\hat{Y}_{N+i \mid N+i-1}=\hat{Y}_{N+i \mid 1,2, \ldots, N+i-1} \\
=E\left\{Y_{N+i} \mid \chi_{N+i-1}, \boldsymbol{\theta}\right\}
\end{gathered}
$$

where $\chi_{N+i-1}$ is the information contained in $\left\{Y_{0}, Y_{1}, \ldots, Y_{N+i-1}\right\}$.

$$
\begin{aligned}
\hat{Y}_{N+i \mid N+i-1}= & \hat{\varphi}_{0}+\hat{\varphi}_{1} Y_{N+i-1}+\hat{\varphi}_{2} Y_{N+i-2}+\left[\hat{\theta}_{0}+\hat{\theta}_{1} Y_{N+i-1}+\hat{\theta}_{2} Y_{N+i-2}\right][1 \\
& \left.-\exp \left\{-\hat{\gamma}\left(Y_{N+i-2}-\hat{c}\right)^{2}\right\}\right]
\end{aligned}
$$

Hence, the one-step ahead forecast error variance is

$$
\sigma_{N+i \mid N+i-1}^{2}=\sigma_{N+i \mid 1,2, \ldots, N+i-1}^{2}=E\left[\left\{\left(Y_{N+i}-\hat{Y}_{N+i \mid N+i-1}\right)^{2} \mid \chi_{N+i-1}\right\}\right]
$$

Formulae for two-step ahead out of sample forecasts are derived analytically by successive use of conditional expectation. For two-step ahead optimal forecast the conditional expectation of $Y_{N+i+1}$ is given by

$$
\begin{gathered}
\hat{Y}_{N+i+1 \mid N+i-1}=\widehat{Y}_{N+i+1 \mid 1,2, \ldots, N+i-1}=E\left[\left\{Y_{N+i+1} \mid \chi_{N+i-1}, \boldsymbol{\theta}\right\}\right] \\
=E\left[E\left[Y_{N+i+1} \mid \chi_{N+i}, \boldsymbol{\theta}\right] \mid \chi_{N+i-1}, \boldsymbol{\theta}\right] \\
=\hat{\varphi}_{0}+\hat{\varphi}_{1} \hat{Y}_{N+i \mid N+i-1}+\hat{\varphi}_{2} Y_{N+i-2}+\left[\hat{\theta}_{0}+\hat{\theta}_{1} \hat{Y}_{N+i \mid N+i-1}+\hat{\theta}_{2} Y_{N+i-2}\right][1 \\
\left.-\exp \left\{-\hat{\gamma}\left(Y_{N+i-2}-\hat{c}\right)^{2}\right\}\right]
\end{gathered}
$$


The two-step ahead forecast error variance is

$$
\begin{aligned}
& \sigma_{N+i+1 \mid N+i-1}^{2}=\sigma_{N+i+1 \mid 1,2, \ldots, N+i-1}^{2} \\
& \quad E\left[E\left\{\left(Y_{N+i+1}-\hat{Y}_{N+i+1 \mid 1,2, \ldots, N+i-1}\right)^{2} \mid \chi_{N+i}\right\} \mid \chi_{N+i-1}\right]+V\left[E\left\{\hat{Y}_{N+i+1 \mid 1,2, \ldots, N+i} \mid \chi_{N+i-1}\right\}\right] \\
& =E\left[\sigma_{N+i+1}^{2} \mid \chi_{N+i-1}\right]+V\left[\left\{\hat{\varphi}_{0}+\hat{\varphi}_{1} Y_{N+i}+\hat{\varphi}_{2} Y_{N+i-2}+\left[\hat{\theta}_{0}+\hat{\theta}_{1} Y_{N+i}+\hat{\theta}_{2} Y_{N+i-2}\right][1-\right.\right. \\
& \left.\left.\left.\exp \left\{-\hat{\gamma}\left(Y_{N+i-2}-\hat{c}\right)^{2}\right\}\right]\right\} \mid \chi_{N+i-1}\right], \\
& =E\left[\sigma_{N+i+1}^{2} \mid \chi_{N+i-1}\right]+V\left[\hat{\varphi}_{1} Y_{N+i}+\hat{\theta}_{1} Y_{N+i}\left[1-\exp \left\{-\hat{\gamma}\left(Y_{N+i-2}-\hat{c}\right)^{2}\right\}\right] \mid \chi_{N+i-1}\right] \\
& \quad=\sigma_{N+i+1}^{2}+\left[\hat{\varphi}_{1}+\hat{\theta}_{1}\left[1-\exp \left\{-\hat{\gamma}\left(Y_{N+i-2}-\hat{c}\right)^{2}\right\}\right]^{2} \sigma_{N+i}^{2}\right.
\end{aligned}
$$

For out-of-sample forecasting, parameters are replaced by their corresponding estimates. By using the above procedure one-step optimal forecasts are computed. To validate whether the formula was providing reasonable forecast, the errors were tested for randomness using run test available at $\mathrm{R}$ software package. The test statistics for sunspot numbers and Global mean temperature was calculated as 0.148 and -1.024 respectively with 0.88 and 0.31 as the corresponding $\mathrm{p}$ value. So, we can infer that the error series were found to be random for both the datasets thereby implying that the model was able to explain the data in an appropriate manner.

\section{Conclusions}

In this present investigation, methodology for fitting ESTAR nonlinear time-series model through Genetic Algorithm methodology is described. It is suggested that, for modelling and forecasting cyclical time-series data, researchers should apply this model rather than the ARIMA model. Given the importance of the issue of global warming, the methodology is employed for modelling sunspot numbers and global temperature datasets, which have been captured by the model employed. Further, optimal forecast formulae have also been derived. As a future work, effort can also be required to be directed towards considering more parsimonious subset 
ESTARMA models. In addition, possibility of employing Fuzzy technique to estimate parameters can also be explored.

\section{Author Declarations:}

Conflicts of interest: Authors state no known conflict of interest.

Availability of data and material: Global temperatures were obtained from the National Aeronautics and Space Administration's (NASA), Goddard Institute for Studies (GISS), while the sunspot numbers were obtained from the Solar Influences Data Analysis Centre.

Code availability: Code will be made available as per requirements.

Author's contribution: Manuscript Preparation: Bishal Gurung, Achal Lama and K N Singh; Data collection and analysis: Bishal Gurung, Achal Lama and Santosha Rathod; Results and Discussion: Bishal Gurung and Achal Lama.

Acknowledgement: Authors are thankful to National Aeronautics and Space Administration, Goddard Institute for Space Studies (https://data.giss.nasa.gov/gistemp) and SIDC-Solar Influence Data Analysis Centre (http://sidc.oma.be/silso) for making the data freely available.

\section{Ethics declarations:}

The authors did not receive financial support from any organization for the submitted work. The authors declare they have no financial interests.

Consent to participate: Not applicable

Consent for publication: Not applicable

\section{References}

1. Baragona R, Battaglia F, Cucina D (2004) Estimating threshold subset autoregressive moving-average models by genetic algorithms. Metron 62:39-61.

2. Boero G, Marrocu E (2004) The performance of SETAR models; a regime conditional evaluation of point, interval and density forecasts. International Journal of Forecasting 20:305-320.

3. Citakoglu H, Babayigit B, Haktanir NA (2020) Solar radiation prediction using multigene genetic programming approach. Theoretical and Applied Climatology 142:885-897 https://doi.org/10.1007/s00704-020-03356-4

4. Fan J, Yao Q (2003) Nonlinear time series: Nonparametric and parametric methods. Springer, New York.

5. GISTEMP Team (2018) GISS Surface Temperature Analysis (GISTEMP). NASA Goddard Institute for Space Studies. Dataset accessed 20YY-MM-DD at https://data.giss.nasa.gov/gistemp/. 
6. Gooijer JGD, Bruin PT (1998) On forecasting SETAR process, Statistics and Probability letters 37:7-14.

7. Gurung B (2013) Modelling and forecasting of volatile time-series data: An application of Genetic Algorithm. International Journal of Agricultural and Statistical Sciences 9:8794.

8. Gurung B, Sarkar KP, Singh KN, Lama A (2021) Modelling annual maximum temperature of India: a distributional approach. Theoretical and Applied Climatology. https://doi.org/10.1007/s00704-021-03674-1

9. Haghbin H, Nematollahi AR (2013) Likelihood-Based Inference in Autoregressive Models with Scaled t-Distributed Innovations by Means of EM-Based Algorithms. Communications in Statistics: Simulation and Computation 42:2239-2252.

10. Hansen J, Ruedy R, Sato M, Lo K (2010) Global surface temperature change. Reviews of Geophysics 48: RG4004, doi:10.1029/2010RG000345.

11. Hassani H, Huang X, Gupta R, Ghodsi M (2016) Does sunspot numbers cause global temperatures? A reconsideration using non-parametric causality tests. Physica A: Statistical Mechanics and its Applications 460:54-65.

12. Merkenschlager C, Koller S, Beck C, Hertig E (2021) Assessing local daily temperatures by means of novel analog approaches: a case study based on the city of Augsburg, Germany. Theoretical and Applied Climatology 145:31-46. https://doi.org/10.1007/s00704-021-03605-0

13. Niglio M (2007) Multi-step forecasts from threshold ARMA models using asymmetric loss functions. Statistical methods and applications 16:395-410.

14. Sivanandam SN, Deepa SN (2008) Introduction to Genetic Algorithms. Springer, New York.

15. Taki M, Rohani A, Yildizhan H (2021) Application of machine learning for solar radiation modeling. Theoretical and Applied Climatology 143:1599-1613. https://doi.org/10.1007/s00704-020-03484-x

16. Terasvirta T (1994) Specification, estimation, and evaluation of smooth transition autoregressive models. Journal of the American Statistical Association 89:208-218. 
17. Toivonen HT (2003) State-dependent parameter models of nonlinear sampled-data systems: A velocity-based linearization approach. International Journal of Control 76:823-32.

18. Woodward WA, Gray HL (1978) New ARMA models for Wolfer's sunspot data. Communications in Statistics: Simulation and Computation 7:97-116.

19. Wu B, Chang CL (2002) Using genetic algorithms to parameters (d, r) estimation for threshold autoregressive models. Computational Statistics and Data Analysis 38:315-330.

20. Young PC, McKenna P, Bruun J (2001) Identification of nonlinear stochastic systems by state dependent parameter estimation. International Journal of Control 74:1837-57. 